\title{
Dynamic imaging of a small artery underneath skin surface of a human finger by optical coherence tomography
}

\author{
Masato Ohmi, Mitsuo Kuwabara, Masamitsu Haruna \\ Division of Health Sciences, Graduate School of Medicine, Osaka University, Suita City, Japan \\ Email: ohmi@sahs.med.osaka-u.ac.jp
}

Received 16 January 2013; revised 21 February 2013; accepted 28 February 2013

\begin{abstract}
OCT is a powerful tool for detection of physiological functions of micro organs underneath the human skin surface, besides the clinical application to ophthalmology, as recently demonstrated by the authors' group. In particular, dynamics of peripheral vessels can be observed clearly in the time-sequential OCT images. Among the vascular system, only the small artery has two physiological functions both for the elastic artery and for muscle-controlled one. It, therefore, is important for dynamic analysis of blood flow and circulation. In the time-sequential OCT images obtained with 25 frames/sec, it is found that the small artery makes a sharp response to sound stress for contraction and expansion while it continues pulsation in synchronization with the heartbeats. This result indicates that the small artery exhibits clearly the two physiological functions for blood flow and circulation. In response to sound stress, blood flow is controlled effectively by thickness change of the tunica media which consists of five to six layers of smooth muscles. It is thus found that the thickness of the tunica media changes remarkably in response to external stress, which shows the activity of the sympathetic nerve. The dynamic analysis of the small artery presented here will allow us not only to understand the mechanism of blood flow control and also to detect abnormal physiological functions in the whole vascular system.
\end{abstract}

Keywords: Optical Coherence Tomography (OCT); Dynamic OCT; Small Artery; Tunica Media;

Sympathetic Nerve; Vascular System

\section{INTRODUCTION}

Since the proposal of optical coherence tomography (OCT) in 1991 [1], OCT has been developed and used intensively for clinical diagnoses of retina diseases in ophthalmology and arteriosclerosis in circulatory medicine [2]. Besides the clinical applications, OCT was applied for brain science, in which the nerve response was detected by the OCT signals [3]. Recently, we demonstrated the dynamic OCT of skin physiology including the dynamics of sweat glands and peripheral vessels was tracked by time-sequential OCT images [4-6]. We discussed in detail mental sweating where mental or physiccal stress was applied to a subject to accelerate excess sweating. When a sound stress was used, we found internal mental sweating without ejection of excess sweat to the skin surface for evaluation of the activity of the sympathetic nerve [7]. On the other hand, the dynamic analysis of peripheral vessels in response to external stress has not been studied yet.

In this paper, we present dynamic analysis of peripheral vessels of a human finger in response to external mental stress. In the vascular system, only a small artery has two different functions both for the Optical coherence tomography (OCT); Dynamic OCT; Small artery; Tunica media; Sympathetic nerve; vascular system elastic artery and for muscle-controlled one, where it expands or contracts in response to external stimulus while a continuous pulsation is made in synchronization with the human heartbeats. Such an attractive motion of the small artery was observed clearly in the second joint of the third finger of a subject by use of the 1.3- $\mu \mathrm{m}$ sweptsource OCT (SS-OCT) imaging system. The dynamic OCT analysis presented here will be useful not only for understanding of the mechanism of the blood flow control but also for detection of abnormal functions and responses of the whole human vascular system.

\section{IMPORTANCE OF THE SMALL ARTERY IN DYNAMIC ANALYSIS OF THE VASUCULAR SYSTEM}

First, we describe here importance of a small artery in dynamic analysis of physiological functions of the vas- 
cular system. In response to external stress, peripheral vessels expand or contract to adjust the blood flow, blood pressure and the skin temperature under control of the sympathetic nerve. Expansion and contraction are made by smooth muscles in the tunica media of the vessel wall, and therefore, the peripheral vessels including small arteries and arterioles belong to the muscle-controlled artery. On the other hand, main and middle arteries make a pulsation in synchronization with the heartbeats by inner and outer elastic films of the vessel wall. The main and middle arteries, therefore, belong to the elastic artery, and these arteries do not make a response to external stress.

It can be seen in a textbook of physiology that a small artery possesses two physiological functions, as described above, both for the elastic artery and for musclecontrolled one [8]. In other words, the small artery makes a continuous pulsation in synchronization with the heartbeat, and expands or contracts in response to external stress. The mechanism of blood-flow control will thus be understood by in vivo OCT observation of dynamics of the small artery. The dynamic observation will also be used for finding of abnormal functions and responses of the whole vascular system.

\section{MATERIALS AND METHODS}

\subsection{In-Vivo OCT Image of a Small Artery of a Human Finger}

The swept-source OCT (SS-OCT: Thorlabs OCM 1300SS) is used for the experiment. The SS-OCT has the coherence length is $12 \mu \mathrm{m}$ with 25 frames/s at the center wavelength of $1.325 \mu \mathrm{m}$. The laser power of the sample arm is $4 \mathrm{~mW}$. The real-time imaging is thus possible by time-sequential OCT images. In our experiment, in vivo OCT imaging of peripheral vessels has been made in the region near the proximal interphalangeal (PIP) joint of the third finger of a volunteer, because all peripheral vessels distribute within the depth of $3 \mathrm{~mm}$ underneath the skin surface. Insertion of a 2-mm-thick glass plate behind an objective brings nearly 1-mm shift of the focal plane at the sacrifice of reduction of the image resolution due to undesirable aberration. Using this sort of simple method for shift of the focal plane, clear OCT imaging is performed successfully near the PIP joint of the third finger of a volunteer (a 23-year-old male), as shown in Figure 1. In the experiment, the finger is pressed against the glass plate with a pressure of $50 \mathrm{mmHg}$. The small artery is imaged to be elliptic shape under this condition. Clear OCT imaging of the small artery is then performed by the existent SS-OCT. The OCT image of the small artery of the third finger is shown in Figure 1, in which the artery becomes elliptic under the pressure. A small artery, as a matter of course, has a circular cross-section

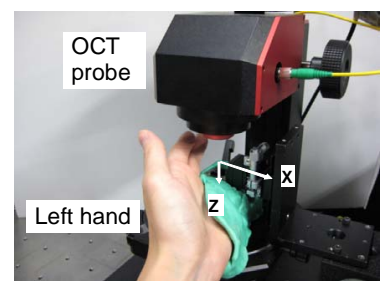

(a)

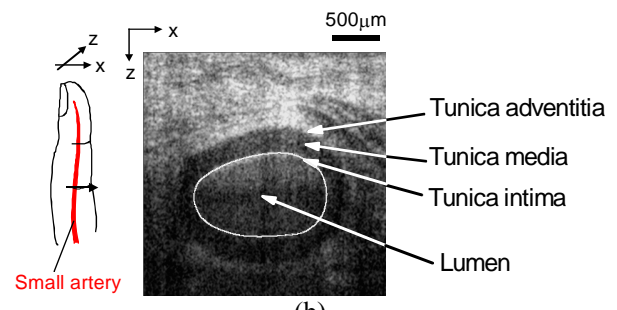

(b)

Figure 1. In vivo OCT image of a small artery underneath the skin surface of a human finger. (a) Photograph of OCT probe where left hand of the subject is fixed with the sample probe; (b) OCT image of a small artery in which there are three layers around the small artery which consist of tunica intima, tunica media and tunica adventitia.

of $1 \mathrm{~mm}$ or more in diameter with the blood pressure of 30 to $70 \mathrm{mmHg}$ [9]. In the actual OCT imaging, the PIP joint of the third finger is pressed against a 2-mm-thick glass plate behind the objective. The artery deforms to be elliptic under the pressure where the minor axis is reduced to $200 \mu \mathrm{m}$ or less. There are three layers around the small artery which consist of tunica intima, tunica media and tunica adventitia. In particular, the tunica media is recognized to be a black band in the OCT image, as shown in Figure 1. The tunica media is composed of five to six muscle layers. In our previous experiment, we have observed in vivo pulsation of the artery in synchronization with the heart beat. Even under the pressure, we have found a phenomenon that the tunica media thickness of small artery was invariant during the pulsation. [5].

\subsection{Time-Sequential OCT of Response of a Small Artery to the Sound Stimulus}

In the experiment, sound stress is applied to the subject after confirmation of the resting state. Here, the stress is unpleasant sound (that is sound of breaking glass) for 0.5 sec at a sound level of $90 \mathrm{~dB}$. The subject is astonished by this sort of sound stress, and behaves as if it defends itself from the enemies. In the physiological response, blood flow into skeletal muscles increases abruptly for the defense, while the small artery of the finger contracts to decrease blood flow. This is a typical defensive response to an astonishing stress, which is one of signifi- 
cant functions of the vascular system.

The response of the small artery to sound stress was tracked by time-sequential OCT images with 25 frames/sec. Dynamic observation of the small artery of human finger is thus possible. Figure 2 shows a time-sequential OCT images of the small artery every five seconds. The sound stress is applied to the subject at $\mathrm{t}=30 \mathrm{~s}$. Immediately after the astonishing sound, the lumen of the small artery contracts gradually, and the cross section of the lumen becomes minim around $t=40 \mathrm{~s}$. It then expands gradually to recover the resting state.

In three thousand time-sequential OCT images obtained with 25 frames/sec, we could measure the crosssectional area of the small artery as well as the thickness of the tunica media. The measurement results are shown in Figure 3. From the results of the variation of the

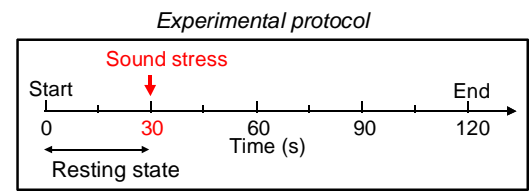

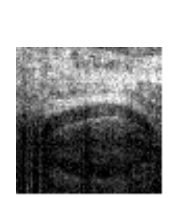

$20 \mathrm{~s}$
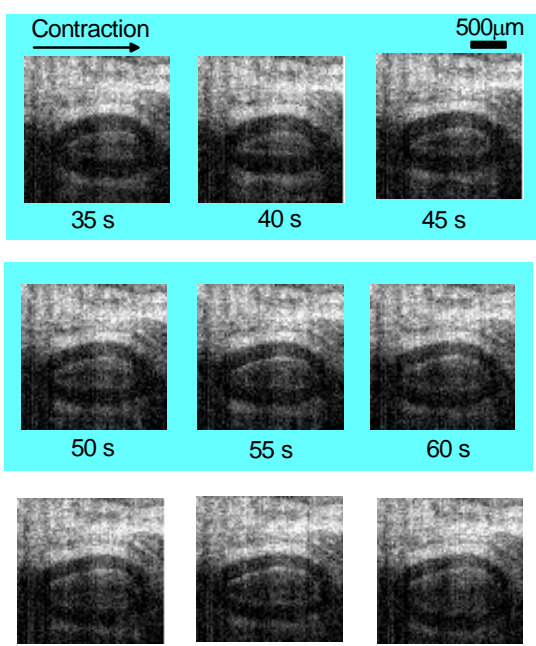

$65 \mathrm{~s}$

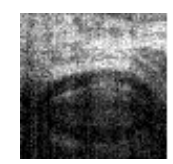

$80 \mathrm{~s}$

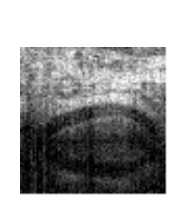

$25 \mathrm{~s}$

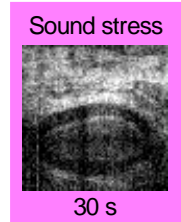

s
Figure 2. Experimental protocol and timesequential OCT images of the small artery with the dynamic motion in response to sound stimulus, where the blue area show the period of construction of the small artery.
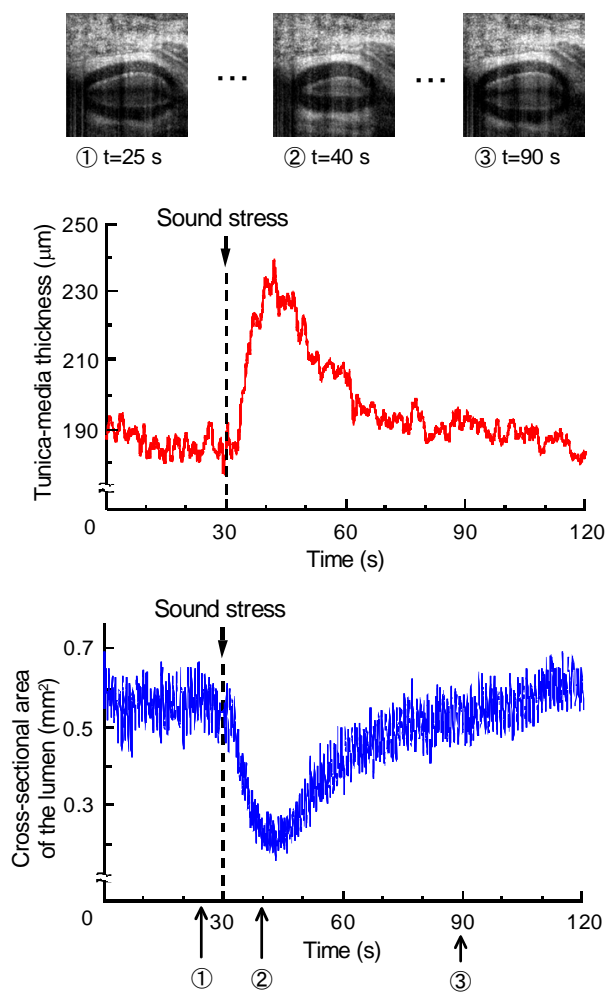

Figure 3. Time variation of the area of the small artery and the thickness of the tunica media in response to sound stimulus.

cross-sectional area of the lumen, it is found that the small artery makes a sharp response to the sound stress while it continues pulsation in synchronization with the heartbeats. The thickness of the tunica media changes abruptly in response to sound stress, where the thickness increases or decreases to contract or expand the small artery, respectively. It is here noted that the tunica media responds only to sound stress, independent of pulsation of the small artery. This means that the tunica media is controlled by the sympathetic nerve.

\section{DISCUSSION AND CONCLUSIONS}

We have demonstrated the dynamic analysis of the small artery of a human finger in response to sound stress. It is found that the small artery contracts and expands in response to sound stress while it continues pulsation in synchronization with the heartbeat. This result indicates that the small artery exhibits two different physiological functions for the elastic artery and the muscle-controlled one. The dynamic OCT of the small artery, therefore, will allow us not only to understand the mechanism of blood flow control and also to find abnormal physiological functions in the whole vascular system.

The velocity distribution in the small artery is being measured using the Doppler OCT [10]. The measurement result should be useful for premonitory symptoms 
of arteriosclerosis and aging of blood vessels. Our effort is directed at comparative study of the dynamic analysis of the small artery and the Doppler OCT.

\section{REFERENCES}

[1] Huang, D., Swanson, E.A., Lin, C.P., Schuman, J.S., Stinson, W.G., Chang, W., Hee, M.R., Flotte, T., Gregory, K., Puliafito, C.A. and Fujimoto, J.G. (1991) Optical coherence tomography. Science, 254, 1178-1181. doi:10.1126/science.1957169

[2] Chen, J. and Lee, L. (2007) Clinical applications and new developments of optical coherence tomography: An evidence-based review. Clinical and Experimental Optometry, 90, 317-335. doi:10.1111/j.1444-0938.2007.00151.x

[3] Maheswari, R.U., Takaoka, H., Homma, R., Kadono, H. and Tanifuji, M. (2002) Implementation of optical coherence tomography (OCT) in visualization of functional structures of cat visual cortex. Optics Communications, 202, 47-54. doi:10.1016/S0030-4018(02)01079-9

[4] Haruna, M., Fuji, T., Ohmi, M. and Hayashi, N. (2006) In Vivo Dynamic Imaging of Arterioles of Human Fingers Using Optical Coherence Tomography at $1.3 \mu \mathrm{m}$. Japanese Journal of Applied Physics, 45, L891-L893.

doi:10.1143/JJAP.45.L891
[5] Kuwabara, M., Fuji, T., Ohmi, M. and Haruna, M. (2008) Dynamic optical coherence tomography of small arteries and veins of human fingers. Applied Physics Express, 1, 058001. doi:10.1143/APEX.1.058001

[6] Saigusa, H., Ueda, Y., Yamada, A., Ohmi, M., Ohnishi, M., Kuwabara, M. and Haruna, M. (2008) Maximum-Intensity-Projection Imaging for Dynamic Analysis of Mental Sweating by Optical Coherence Tomography. Applied Physics Express, 1, 098001. doi:10.1143/APEX.1.098001

[7] Ohmi, M., Tanigawa, M., Yamada, A., Ueda, Y. and M. Haruna, M. (2009) Dynamic analysis of internal and external sweating by optical coherence tomography. Journal of Biomedical Optics, 14, 014026. doi:10.1117/1.3079808

[8] Burkitt, H.G. (1993) Wheater's functional histology, Churchill, Livingstone, 162.

[9] Mizoguti, H. (1987) A textbook and Atlas of histology. 2nd Edition, Kanehara, Japan, 131.

[10] Yang, V.X.D., Gordon, M., Qi, B., J. Pekar, J., Lo, S., Seng-Yue, E., Mok, A., Wilson, B. and Vitkin, I. (2003) High speed, wide velocity dynamic range Doppler optical coherence tomography (Part II): Imaging in vivo cardiac dynamics of Xenopus laevis. Optics Express, 11, 16501658. doi:10.1364/OE. 11.001650 